\title{
Kurzbeiträge
}

\author{
Frank Schulz-Nieswandt
}

\section{M.A.U.S.S. spielen! Die Gabe im multi-disziplinären Blick - Über den zentralen anthropologischen Baustein der Reziprozität und Mutualität als kulturgrammatische Prinzipien der genossenschaftlichen Gestalt}

\section{Vorbemerkungen}

Der Beitrag (als Typus einer post-strukturalistischen Argumentation) legitimiert sich in der vorliegenden Positionierung einerseits durch das Erkenntnisinteresse, genossenschaftliche Sozialgebilde hermeneutisch zu erschließen (Schulz-Nieswandt 2006), indem - betriebsmorphologisch - die Logik der Gebilde als Programmcode de-chiffriert wird (Schulz-Nieswandt 2007 a). Dabei argumentiere ich (kultursemiotisch: Schulz-Nieswandt/Sauer 2010) im epistemischen Raum einer trans-utilitaristischen Theoriebildung, die den nutzentheoretischen Reduktionismus vermeidet. ${ }^{1}$ In diesem Sinne gründete Caillé 1981 die M.A.U.S.S.-Bewegung (Mouvement AntiUtilitariste dans les Sciences Sociales), die den ökonomi(isti)schen Utilitarismus der Nutzentheorie in den Sozialwissenschaften kritisiert (vgl. auch Caillé 2008).

Das Genossenschaftliche als Formprinzip wird hierbei nicht primär im Rahmen einer Rechtsmorphologie verstanden, sondern als soziales Gebilde, dass von der Logik der Gabe sowie der Praxis der Gegenseitigkeit der Hilfe geprägt ist. Genossenschaftlichkeit meint das Strukturprinzip von Sozialgebilden, freiwillig und gewollt eine Hilfe auf Gegenseitigkeit zu praktizieren, also die Mutualität der Hilfe von Mitgliedern einer Gemeinschaft förderwirtschaftlich auf der Basis eines Reziprozitätsprinzips, das nicht unbedingt vom Typus einer Leistungsäquivalenz geprägt sein muss, sondern solidarischer Natur ist, zu betreiben.

Die Kommune als die die Genossenschaft einbettende Kontextualität wird im Sinne dieses genossenschaftlichen Formprinzips entfaltet als vernetzter Sozialraum des gelingenden sozialen Miteinanders angesichts der Differenz von Identität und Alterität in der dialektischen reziproken Beziehung.

1 Dies ist auch in anderen (sozialpolitischen: Schulz-Nieswandt 2013 c; 2013 d; 2013 f) Forschungsfeldern fruchtbar anzuwenden. 


\section{Fundamentalontologische Grammatik des Problems}

Andererseits wird dadurch die Normativität der sozialen Gebilde, im Sinne eines ontologischen Wahrheitsverständnisses, ${ }^{2}$ nach der die Form der Genossenschaft $\mathbf{g F}$ eine generative Bedeutung hat für die Form als Ausdrucksform (aF) des gelingenden personalen Selbst-Sein als Daseinsqualität $\Omega$ erhält, begründet:

$$
\mathbf{g F} \rightarrow \mathrm{aF}(\Omega) .
$$

$\Omega$ ist, letztendlich onto-theologisch gesehen, das gelingende soziale Miteinander der Personalität im Formmodus des Genossenschaftlichen, das als Gebilde uno actu performativer Ausdruck (aF) und generatives Prinzip (gF) ist, also Präsenz als Selbst-Inszenierung und Skript als Programmcode der Inszenierung zugleich. Anders ausgedrückt: Es geht um eine fundamentale Neuformulierung der in der Genossenschaftswissenschaft lange schon kontrovers diskutierten Idee des homo cooperativus (vgl. in Blome-Drees 2011; Brinkmann 2010) im Rahmen ${ }^{3}$ einer nicht-trivialen Form-Inhalts-Metaphysik, wonach die Form F eben nicht einfach die Form eines Inhalts I - also $\mathrm{F}=\mathrm{F}(\mathrm{I})-$ wäre.

Dagegen gilt es, nochmals formuliert, zu argumentieren: Die Form transportiert $\Omega$ als Ausdrucksform aF, aber in dieser Präsenz der Inszenierung nur, weil F als gF uno actu schöpferische Kraft dieser performativen Praxis ist.

Das genossenschaftliche Formprinzip ist somit Ei und Henne zugleich. Mit dieser Aporie muss die Sozialtheorie leben. Das Ei ist eine Funktion der Henne wie auch die Henne eine Funktion des Ei ist. ${ }^{4}$ Obwohl $\mathrm{E} \neq \mathrm{H}$ ist, gilt:

$$
E=f(H) \text { und } H=f(E) .
$$

2 Was aber hier hinreichend nicht (Aspekte dazu auch in Schulz-Nieswandt/Köstler 2012; vorher in Schulz-Nieswandt 2006) geleistet werden kann.

3 Es gibt natürlich auch andere Wege zum Thema, etwa die der Soziobiologie (Voland 2013) oder der evolutorischen Kooperationsforschung (Kappeler/Schaik 2005). Aber dann wäre vor allem auch an Peter Kropotkin (1842-1921) zu erinnern: vgl. Heinz 1989.

4 Mag die Analyse auch zunächst soziologisch nach den notwendigen Entwicklungsressourcen (Vertrauens-, Sozial- und Humankapital) fragen, um der sozialen Produktionsfunktion des Prozessgeschehens auf die Spur zu kommen. Alsbald ist dann die Ebene der Ethik und Pädagogik des Sozialcharakters der Person (,Auf die Haltung kommt es an!") erreicht. Und damit zeichnen sich die grundlegenden Fragen nach der Möglichkeit gelingender Gesellschaft auf personaler Grundlage ab. Vertrauen reduziert Unsicherheit und ermöglicht Netzwerke des Miteinanders und produziert so das Sozialkapital der „Kooperationsgewinne“ (eine Teilmenge von Pareto-Lösungen, die der von Kant geprägte Rechtsphilosoph John Rawls als Fairness dargelegt hat). Allerdings setzt die Bildung von Vertrauenskapital auch wiederum bereits das Leben von Netzwerken voraus, denn nur in den konkret gelebten/ praktizierten Netzwerken kann sich das Vertrauensklima zwischen den Mitgliedern de facto entwickeln. Die Soziologie der Netzwerke hat also tatsächlich das bekannte und ebenso ungeliebte Problem von Henne und Ei vor sich liegen. Ein Miteinander setzt Vertrauen voraus; Vertrauen bildet sich erst im Miteinander. Deshalb benötigt Netzwerkbildung ein Vertrauensvorschuss. Und Netzwerke brauchen eine gewisse Dauer der Reifung. Vertrauensbildung benötigt Zeit, ist also eine Funktion gelingenden Netzwerkerlebens. Um den Kreislauf von Vertrauenskapitalbildung und Sozialkapitalgenerierung in Gang zu setzen, benötigt der Prozess somit in der Ausgangslage bereits personale Haltungen, die von einer gelebten positiven Anthropologie des Vertrauensvorschusses, der Geduld, der längeren Zeithorizonte, einer rechten Mischung aus Eifer und Gelassenheit, von Erwartungsanspruch und Vergabe-Bereitschaft im Fall von Scheiternsrisiken und Entwicklungskrisen gekennzeichnet ist. So wurzelt der gelingende kumulative Kreislauf von Sozialkapital und Vertrauenskapital in einem transzendental vorgängigen Human(start)kapitals. Personale Haltungen sind in diesem Sinne eine (in diesem Sinne: transzendentale) Voraussetzung der Möglichkeit gelingenden sozialen Miteinanders. Auf den Sozialcharakter kommt es an. 
Kern dieser generierten Performativität ist die Gabe. Die Gegen-Gabe GG kann nur eine Funktion einer vorgängigen Gabe $\mathrm{G}$ sein, die (transzendental gedacht) unbedingt ist; erst im Zyklus wird eine zweite Generation von $G$, nämlich $G^{*}$ eine Funktion von GG sein:

$$
\mathrm{G} \rightarrow \mathrm{GG}=\mathrm{f}(\mathrm{G}) \rightarrow \mathrm{G}^{*} \rightarrow \mathrm{GG}^{*} \rightarrow \ldots
$$

Diese transzendentale Unbedingtheit von $\mathrm{G}$ ist empirisch nicht zu denken, aber sie ist sozialtheoretisch im Rekurs auf eine propädeutische philosophische Anthropologie denknotwendig. So ist, daseinsanalytisch gesehen und fundamentalontologisch formuliert, jeder in aller Entschlossenheit entworfene Selbst-Entwurf der Person in ihrer Eigentlichkeit zugleich immer ein geworfener Entwurf, der angesichts der Endlichkeit, die die Freiheit erst ermöglicht, sich der Vorgängigkeit einer nicht selbst verfügten Gabe verdankt. So verdankt sich der Mensch in seinem personalen Sein der ,zweiten, sozio-kulturellen Geburt“ (Dieter Claessens), die immer in vorgängigen konkreten sozialen Lernfeldern geschieht, in die eben diese Personalisierung transaktional (dazu der personenzentrierte [Personalismus meint mehr und anderes als Personologismus] Ansatz in Schulz-Nieswandt 2006 a; Schulz-Nieswandt 2007 a; 2008) eingebunden ist.

Die Gabe ist in ihrer Unbedingtheit einerseits schöpferischer Kern der dynamischen Mechanismen der Reziprozität, die den Modus der solidarischen Mutualität annehmen kann, sie ist andererseits dann aber auch integrierter Teil (der Textur) des Sozialen, wobei Gesellung und wirtschaftlicher Zweck (Austausch von Ressourcen) zusammenfallen, also eine kooperative Art der moralischen Ökonomik darstellt.

Insofern haftet der Gabe immer eine ökonomische Dimension an, da Ressourcen in den Mechanismus von Gabe und Gegen-Gabe eingehen. Damit wird die Gabe aber nicht automatisch von einer Ökonomik des Nutzens dominiert; auch der Markttausch bleibt nur ein Typus in der Vielfalt (einer formalen Soziologie) der Austauschbeziehungen (Gimesi/Hanselitsch 2010; Mohr/Ouart/ Vetter 2013). Der alte Vorwurf vom Tautologismus in der utilitaristischen homo oeconomicusTheorie wird hier wieder lebendig. Und dies zu Recht: Denn es kommt auf die Motive in den sozialen Beziehungen an, weil davon auch die Qualität der Netzwerke abhängt. Es ist eben nicht egal, wenn, phänomenologisch erschlossen, z. B. helfende Sorge (insbesondere habitualisiert) getragen wird von Liebe, Anerkennung, Respekt, Schuld, Dankbarkeit, Mitleid, Barmherzigkeit und Gnade, von Generosität oder Philanthropie usw., oder auch von demütigender Dominanzstreben, von Macht und herrschaftlichen Kontrollinteressen. Vor allem: Dies sind alles figurative Kategorien, die, sozialontologisch unabdingbar, immer auf den Kontext sozialer Beziehungen verweisen, in denen sie eingelassen sind.

\section{Die kulturelle Grammatik des Genossenschaftlichen}

Das Prinzip der Reziprozität $\mathbf{R}$ des homo reciprocans bezieht sich auf die Transformation der linearen Sequenz von Geben G des homo donans und Nehmen N zur (u. a. aus der römischen Rechtsgeschichte der Korporationen überlieferten) Mutualität $\mathbf{M}$ dann, wenn auch das Nehmen die Gegen-Gabe GG und das Entgegen-Nehmen EN der Gegen-Gabe, und im weiteren Zyklus die erneute $\mathrm{G}^{*}$ folgen:

$$
\mathbf{R}: \mathrm{G} \rightarrow \mathrm{N} \rightarrow \mathrm{M}: \mathrm{GG} \rightarrow \mathrm{EN} \ldots \rightarrow \mathrm{G}^{*} \rightarrow \ldots
$$

Damit bleibt Marcel Mauss (Moebius 2006) der zentrale Klassiker dieser Denkrichtung. 
Genossenschaften sind nun, wie bereits eingangs definiert, selbstorganisierte, selbstverwaltete Sozialgebilde auf der Basis der Gegenseitigkeitshilfe, als Bedarfsdeckung der Mitglieder auf Gegenseitigkeit. Das ist kulturgeschichtlich ubiquitär (Schulz-Nieswandt 2003), etwa auch entfaltet in der Gildenforschungen der 1970er und 1980er Jahre in Deutschland (Schulz-Nieswandt 2000), u. a. im Kontext von Forschung zum Kommunalismus des europäischen Siedlungswesens im Übergang vom Mittealter zur Neuzeit. ${ }^{5}$ In diesem Sinne ist - entgegen einer öffentlich-rechtlichen Engführung - auch die GKV (in ihrer sozialökonomischen Morphologie re-distributiver Logik: ${ }^{6}$ Schulz-Nieswandt 2002) in einem Kern eine Risikogemeinschaft von (sequenziellen) NettoEmpfängern und (sequenziellen) Netto-Gebern in einem komplexen inter-temporalen (und eben nicht vollständig äquivalent bilanzierten) Ausgleichsprozess. Strukturgleich ist der Mechanismus des Generationenvertrages zu verstehen (Schulz-Nieswandt u. a. 2009). ${ }^{7}$

Und immer dann, wenn genossenschaftliche Gebilde Aufgaben von quasi öffentlicher Bedeutung aufgreifen und erfüllen, kristallisiert sich eine Gemeinwirtschaftlichkeit heraus (Schulz-Nieswandt 2011 a), die mitunter als funktionale Äquivalenz zur munizipalsozialistischen öffentlichen Wirtschaft (etwa im Kontext der [problematischen: Schulz-Nieswandt 2013b] Privatisierung von Bürgervermögen) zu verstehen ist und die dergestalt wirksam werden kann. Man denke an die Situation der öffentlich-rechtlichen Sparkassen und der Kreditgenossenschaften in Deutschland als „ungleiche Zwillinge“ (Schulz-Nieswandt/Köstler 2012).

\section{Die Konjunktur des Gaben-Themas}

Die Kategorie der Gabe ${ }^{8}$ hat seit Jahren eine enorme Konjunktur. ${ }^{9}$ Und dies nicht nur thematisch. Diese Konjunktur ist auch, in nachfolgenden Darlegungen ohne Anspruch ${ }^{10}$ auf hinreichend erschöpfende Wiedergabe der Literatur diskutiert, daran abzulesen, dass auch für 2014 grundlegende Beiträge zum Thema angekündigt sind (u. a. Grund 2014; Hobuß/Tams 2014), u. a. auch wieder eine Übersetzung von Marcel Hénaff (2014) aus dem Französischen. Und das Thema wird keine temporäre Blase sein. ${ }^{11}$ Tiefe Einsichten sind vielmehr wieder neu entdeckt worden.

5 Wobei u. a. der kommunitaristische Blick diese Sicht auf die Kommunalität des Daseins des Menschen in seinem topographischen Netzwerkgefüge wieder aktualisiert hat: vgl. auch Schulz-Nieswandt/Köstler $2012 \mathrm{a}$.

6 Insgesamt schließt sich gerade hier der Forschungsstrang zur Moralökonomik (moral economy) an, der verschiedene forschungsgeschichtliche Wurzeln hat und in die Analyse des wohlfahrtsstaatlichen Leistungsgeschehens Eingang gefunden hat.

7 Die Frage der Risikomischung bzw. der Vermeidung von Risiko(selbst)selektion ist auch zentral in der Debatte um das berufsgenossenschaftliche Monopol: Schulz-Nieswandt $2011 \mathrm{c}$.

8 Überblick in Adloff 2010 und in Frank 2012.

9 Auch im transnationalen Kontext mit post-kolonialem Blick auf die Ambivalenz der Hilfe in der Entwicklungszusammenarbeit. Vgl. Köstler 2013. Wir haben in Köln die Reziprozitätsforschung in recht unterschiedlichen Themenfeldern an Sozialgebilden der (gegenseitigen) Hilfe angesiedelt. Vgl. etwa Köstler 2012; Köstler/SchulzNieswandt 2010. Ferner mit Bezug auf Wohnformen Schulz-Nieswandt/Köstler/Langenhorst/Marks (2012) sowie in Bezug auf Gesundheitsselbsthilfegruppen Schulz-Nieswandt 2011 sowie 2012 d. Schließlich SchulzNieswandt 2013 a.

10 Auch andere Autoren leisten sich, sogar entgegen einer reflektierten Selbsteinschätzung, erhebliche Lücken in der Literatur- und Materialaufarbeitung, etwa Joas 2014. Dennoch ist sein Beitrag produktiv, vgl. Laux 2013.

11 Nachfolgende Ausführungen führen u. a. Schulz-Nieswandt 2013, S. 68ff. sowie in Schulz-Nieswandt 2010, S. 565ff. fort. Vgl. auch in Schulz-Nieswandt/Köstler 2011, S. 149ff. 


\section{Die aktuelle Konjunktur und ihre lange Wachstumsvorgeschichte}

Von kulturwissenschaftlichen Verhandlungen des Ökonomischen ist die Rede (Mein/Schößler 2005); und die Gabe wird zur Figur des Ursprungs von Kultur (Trinkaus 2005). In recht unterschiedlichen Themenfeldern findet die Gabenforschung Eingang (vgl. etwa auch Motakef 2011 oder Maio 3013).

Es handelt sich quasi um eine Wiedergeburt, war die Gabe - und damit der homo donans - doch eine klassische Kategorie der klassischen Soziologie, vor allem in ihrer frühen Verbindung zur Ethnologie, und dies nicht nur in Frankreich, auch zum Teil in Deutschland. Auch die frühe Rechtsethnologie wäre hier anzufügen. Und der Potlatch bleibt (Schulz-Nieswandt 2001 a) ein Forschungsthema. Das Thema bleibt ferner zentral in der Wirtschaftsethnologie (Lang 2010; Rössler 2005), wenngleich es dort (Görlich 1992; Znoj 1995) m. E. nicht immer angemessen transutilitaristisch behandelt wird; ebenso bleibt das Thema relevant bei der Suche von lokalen (wohl nicht generalisierbaren) Alternativen zur Geldwirtschaft (Preissing 2009; Thiel 2011), womit Phänomene wie Tauschringe (Köstler 2007; vom Ertrag her gesehen eher dünn: Wagner 2008) oder Regionalwährungen angesprochen sind. Auch patriarchatskritische Rezeptionskontexte liegen ferner vor (Wörer 2012). Die spezifische rechtsgeschichtliche Genossenschaftsforschung (Otto von Gierke [1841-1921] vgl. auch in Schulz-Nieswandt 2003) in Deutschland findet hierbei ihre Wahlverwandtschaften in der solidaristischen Schule von Ėmile Durkheim (1858-1917).

Ich möchte hier nun nicht nochmals, wie es in einer mehr oder weniger guten Sekundärliteratur der neueren Zeit geschah, die Reihe der Klassiker vorstellen: Nur zu nennen sind nochmals Marcel Mauss (1872-1950), Bronislaw K. Malinowski (1884-1942), Richard Turnwald (1869-1954), zum Teil Georg Simmel (1858-1918), dann auch Karl P. Polanyi (1886-1964), Marshall Sahlins (*1930), später dann auch Maurice Godelier (*1934) und Paul Ricoeur (1913-2005), der frühe Pierre Bourdieu (1930-2002), in seiner spezifischen Art auch Claude Lévi-Strauss (1908-2009). Viel diskutiert werden die neueren Beiträge von Alain Caillé (*1944) und Marcel Hénaff (Hénaff 2009), wenngleich ${ }^{12}$ die dort ausformulierten sphärenspezifischen Differenzierungen sowie die Ideen zu einem Strukturwandel in Bezug auf den öffentlichen und privaten Raum auch nicht wirklich neu sind (und z. T. im Kommunitarismus zu finden sind).

Und die soziologische Sekundärliteratur (in Deutschland u. a. von Stephan Moebius, Frank Adloff, Frank Hillebrandt, Christian Stegbauer, Steffen Mau, Fritz Rüdiger Volk u. a., ${ }^{13}$ mit Blick auf die Umverteilungsakzeptanz vgl. auch die Arbeiten von Carsten Ullrich oder von Ursula Dallinger) ist umfassend, ${ }^{14}$ ebenso die eher (ethnologisch informierte) philosophische Sekundärliteratur (etwa wie bei Iris Därmann 2010).

Ich selbst habe die Gabeanthropologie in sozialpolitischer Absicht vor allem in einem strukturalistisch de-chiffrierten Code archetypischer Art nachgezeichnet (Schulz-Nieswandt 2003). Demnach gilt:

12 Die herausragende innovative Bedeutung von Hénaff kann ich auch nach der Lektüre des instruktiven Aufsatzes von Moebius (2010) nicht entdecken.

13 Einige Aufsatzbände sind hier verdienstvoll (Moebius/Papilloud 2006; Adloff/Mau 2005) editiert und kommentiert worden.

14 Nicht so oft zitiert, aber ertragreich: Pulcini 2004. 
Herrschaft : Genossenschaft $=$ öffentliche Speisung ${ }^{15}$ : Mahlgemeinschaft. ${ }^{16}$ Topographisch korreliert dies zur Binärik von

Vertikalität : Horizontalität.

Das Schrifttum wächst noch an, wenn phänomenologisch die Schnittstellen zu solchen Kategorien wie die des Schenkens (vgl. Klein 2010; Stephan 2010; an die älteren ${ }^{17}$ Beiträge von Helmuth Berking, Gerhard Schmied und von Johanna Krafft-Krivanic erinnernd) oder des Stiftens (mit einer Reihe gerade jüngerer Fachbeiträge: vgl. u. a. Adloff 2010) Berücksichtigung findet.

Auch hat sich eine eigene Diskussion um die unschönen, schmutzigen bzw. bösartigen Gaben (bzw. der Gabe-Motive) entwickelt (Starobinski 1994).

An die neuere (daseinsthematische) Forschung über das öffentliche Tafeln als personale Erlebnisgeschehensordnung ist zu erinnern. Es geht um die Diskurse über Demütigung im Wohlfahrtsstaat, eine auch rechtsphilosophisch zunehmend diskutierte Problematik. ${ }^{18}$ Kritisch gesehen werden die Umkipp-Effekte der Hilfe in Form einer neo-liberalen gouvernementalen Selbst-Befähigung zum unternehmerischen Selbst, das vielmehr tatsächlich als „unternommenes“ Subjekt gesellschaftlich dispositiviert ist. In der Forschung zum bürgerschaftlichen Engagement geht es auch um die Frage der Dankbarkeit als Abhängigkeitserfahrung. Die Einwilligung in Abhängigkeit (in der Altenpflegeforschung) wird dagegen mit guten Gründen als Entwicklungsaufgabe im höheren Alter psychodynamisch diskutiert.

Und es ist (in der Tradition der Erforschung des Potlatch-Phänomens [Schulz-Nieswandt 2001a] stehend) auf das Problem destruktiver Gabezyklen (konsum- und überschuldungssoziologisch: Schulz-Nieswandt/Kurscheid 2007) zu verweisen. Eine eigene Diskussion um eine Ökonomie der (Hermeneutik der) Verschwendung (Hoffmann 2009), nicht nur an Thorstein Veblen (1857-1929) orientiert, knüpft hier an (ferner Bähr 2009). Von besonderer Bedeutung ist diesbezüglich bereits das Werk von Georges Bataille (1897-1962). Und an Bataille (1985) knüpft auch Jean Baudrillard (1929-2007) an (Baudrillard 2011), wenn er im Tod den Höhepunkt eines symbolischen Tausches erkennt (Rückgabe des Lebens), einer natürliche Zyklizität von Gabe (Geburt) und Rück-Gabe (Tod), den die Theorie des ökonomistischen Funktionalismus des Kapitalismus nicht versteht, da sie (wie auch die korrespondierende Praxis) eine gegenläufige Logik des linearen Akkumulationsdynamismus vergöttert, wodurch verständlich wird, warum der freiwillige Selbstmord ein öffentlicher Skandal ist, da der Mensch sich das Recht (gegen die Gesellschaft) herausnimmt, sich dem ökonomischen Funktionalismus zu entziehen.

Hinzu kommt noch eine religionswissenschaftliche und theologische Debatte zum Opfer. ${ }^{19}$

Die Forschungsliteratur spannt sich bereits über einen Zeitraum von über 100 Jahren auf. Die neuere, mitunter von Moses I. Finley (1912-1986: Finley 2005) geprägte (insbesondere französische) Altertumsforschung hat Gabe und Reziprozität im archaischen Griechenland erforscht

15 Geöffnet werden hierzu die königlichen Magazine.

16 Eingebettet ist die Mahlgemeinschaft in die kultische Opferpraxis (Schulz-Nieswandt 2001).

17 Weitgehend vergessen scheint der Beitrag von Francois Perroux (1903-1987) zu sein, der neben dem Tausch auch den Zwang und das Schenken in der ökonomischen Analyse berücksichtigt haben wollte. Ebenso gilt diese Vergessenheit wohl für Bernhard Laum (1884-1974) angesichts seiner Theorie der religiösen Ursprünge des Geldes.

18 Ich habe früher schon zwischen dem Archetypus der ekklesiatischen Sozialversicherung und der proskynetischen Sozialhilfe unterschieden: Schulz-Nieswandt 2002.

19 Aus der Fülle der Literatur vgl. auch Negel (2005), der auch eine instruktive Typologie der Opfertheorien liefert. Vgl. auch Berner/Bochinger/Flasche 2005. 
(Wagner-Hasel 2000). An die Euergetismus-Forschung im griechisch-römischen Kulturraum ist zu erinnern, ohne hier Spezialstudien anzuführen. Die Soziologie der homerischen Gesellschaft hat dabei tief greifende Erträge erarbeiten können. Hier konnten vor allem auch familialistischverwandtschaftliche oder gar (z. T. deutschrechtliche) tribale Deutungsmuster der sozialen Gesellung in der polis zugunsten artifizieller Formen des Vereinswesens und der Kultgenossenschaften relativiert werden. Die (ethnologisch, z. T. psychoanalytisch aufgeklärte) französische Altertumsforschung hat auch hier mit der älteren deutschen (tribalistisch orientierten) Forschung aufgeräumt.

Auch Beiträge zur symbolischen Praxis von Gabe-Inszenierungen im europäischen Mittelalter (Algazi/Groebner/Jussen 2003; Reichlin 2009) im Kontext von Politik, Macht, Herrschaft, aber auch von Bünden und Freundschaften haben sich entwickelt (Davis 2002).

\section{Die transgressive Innovativität des non- und transutilitaristischen Denkens}

In der englischsprachigen, vor allem US-amerikanischen Fachdiskussion laufen die Themen u. a. unter der Rubrik ,gift (exchange) economics/theory“. Sie soll hier nicht weiter entfaltet werden, weil sie m. E. keinen theoretischen Mehrwert gegenüber der deutschsprachigen Debatte aufzuweisen hat. ${ }^{20}$ Als herausragend muss die französische Theorietradition genannt werden. So gründete, wie eingangs schon angesprochen, der angeführte Caillé 1981 die M.A.U.S.S.- Bewegung (Mouvement Anti-Utilitariste dans les Sciences Sociales), die den ökonomi(isti)schen Utilitarismus der Nutzentheorie in den Sozialwissenschaften kritisiert (vgl. auch Caillé 2008).

Ich habe immer wieder kritisiert, dass die vor allem soziologische Literatur die Kategorie oftmals - auch in der Wirtschaftssoziologie i. e. S. - stark utilitaristisch rezipiert hat und im Rahmen von (Aus-)Tauschtheorien ${ }^{21}$ vor dem Hintergrund spezifischer Rationalitätsverständnisse gefiltert und der Effizienz-orientierten, weil auf die Allokation von Wohlstandspositionen abstellenden Kategorie der (fairen) Reziprozität subsummiert hat (Scheuregger u. a. 2007). Das gilt für die neuere Mikroökonomik ebenso wie für Rational Choice-Soziologien. Die Reziprozitätsforschung hat dann auch Eingang gefunden in dem komplexen Feld der Netzwerkforschung (Stegbauer/Häußling 2010).

\section{Exkurs: Rationalität, Leiblichkeit und das Unbewusste}

Die ökonomische Theorie in ihrer heute höchsten Entwicklungsstufe ist in der Lage, im Lichte eines Paradigmas der willentlichen Intentionalität das Theorem eines rationalen Altruismus (definiert über Freiwilligkeit und Einstimmigkeit) zu modellieren. Dieses Verhaltensmuster mag auch in Routine übergehen. Es bleibt aber der Modellwelt des Paretianismus verhaftet: Positive

20 Typisch ist hier z. B. Liebersohn 2011.

21 Ich verweise u. a. auf die Theorielinie von Peter Blau (1918-2002). Wichtig sind auch die Beiträge von Alvin W. Gouldner (1920-1980). 
Externalitäten werden als direkte Interdependenz der Nutzenfunktionen dergestalt thematisiert, dass eine Rawlsianische Teilmenge aller Pareto-Lösungen in Form von Kooperationserträgen möglich werden. Es gilt: Maximiere die Soziale Wohlfahrt SW auf der Basis der $\mathrm{U}_{\mathrm{i}}$, also SW $\rightarrow$ max!, wobei (Rawlsianische Pareto-Lösung):

$$
\mathrm{SW}\left(\mathrm{U}_{\mathrm{i}}\right)>0 \text { für alle } \mathrm{i}=1 \ldots \mathrm{n} \text {. }
$$

Jede Rawlsianische Lösung ist demnach eine Pareto-Lösung, aber nicht jede Pareto-Lösung ist auch eine Rawlsianische Lösung. Die Differenz der Definition (der Aggregationslogik) der sozialen Wohlfahrt SW drückt in Bezug auf die individuellen $(i=1 \ldots n)$ Nutzenfunktionen $U_{i}$ aus in

$$
\begin{gathered}
\left\{\partial \mathrm{SW} / \partial \mathrm{U}_{\mathrm{i}}>0 \text { für alle } \mathrm{i}=1 \ldots \mathrm{n}\right\} \\
\quad \neq \\
\left\{\partial \mathrm{SW} / \partial \mathrm{U}_{\mathrm{i}} \geq 0 \text { für alle } \mathrm{i}=1 \ldots \mathrm{n}\right\} .
\end{gathered}
$$

Kennt jede Pareto-Lösung keine absoluten (wohl aber relative) Verlierer, so jede Rawls-Lösung nicht nur nicht keine Verlierer, sondern auch Möglichkeitslösungen für relative gemeinsame Wohlstandsverbesserungen. ${ }^{22}$

So wird die Rationalität in die strategische Verkettung der Akteure transportiert; von der Inkorporierung solcher Prozesse in die unbewussten Tiefen eines dispositiven Habitus ist die Theorie epistemisch strikt abgetrennt. Damit werden auch keine/kaum Anleihen in einer Affektpsychologie (Schulz-Nieswandt 2012 b) oder in einer Soziologie der Emotionen unternommen; die ökonomische Verhaltenswissenschaft öffnet sich zwar der Kognitionspsychologie, auch durch neurowissenschaftliche Methoden unterstützt. Doch die Inter-Disziplinarität ist doch eng angelegt und hinterfragt nicht das rationale Paradigma willentlichen Nutzenstrebens. Das überrascht, da seit der Freud'schen Revolution das Unbewusste nicht mehr zu eskamotieren ist. Und der Mensch ist aus seiner ganzen Leiblichkeit heraus zu verstehen, zwar als Ökonomik der Begierde und des Begehrens, aber viel komplexer, tiefschichtiger (Moebius 2006 a) und vor allem ambivalenter als es im ökonomischen Verhaltensansatz modelliert wird und werden kann.

Aus dem Exkurs zurück, lautet daher die Theoriediagnose: Die non- oder trans-utilitaristischen Traditionen, vor allem dann, wenn sie durkheimianisch (Suber 20119 organisiert sind, werden vorschnell vergessen oder gar übergangen. ${ }^{23}$

22 Es darf hier darauf verwiesen werden, dass das allokative Effizienz- Kriterium im Pareto-Form struktural analog ist zum Sittengesetz bei Kant, so dass sich ergibt:

Pareto : Kant = allokative Effizienz : Ethik im Modus des Sittengesetzes.

Dies verweist $u$. a. auf die oftmals kann nicht erkannte sittliche Tiefenstruktur der ökonomischen Kardinalproblematik. Aber das Problem, das eigentlich bereits auf der Theorie der Sympathie als sittliche Einbettung ökonomischen Handelns bei Adam Smith (1723-1790) dogmengeschichtlich bekannt sein sollte, erweist sich zugleich als strukturale Relationslogik in einem institutionentheoretischen Sinne, denn es gilt:

Moral : Sittlichkeit $=$ Kant : Hegel.

Dies lenkt das Argument der sittlichen Einbettung bei Smith auf die aristotelischen Wurzeln der Tugendethik in ihrer Relevanz auch unter modernen Bedingungen der Vertragsgesellschaft des Marktes. Damit wird nicht nur erneut die anthropologische Reichweite des durkheimianischen Satzes von den nicht-kontraktuellen Voraussetzungen des Kontrakts verständlich; sondern es wird die Entwicklung von Kant (1724-1804) zu Hegel (1770-1831) unter der Schleife zur antiken Tugendphilosophie bei Hegel als Bewegung von der innerlichen Moralität zur institutionellen Einbettung der Moralität als Sittlichkeit deutlich:

Ökonomik : Moralität $=$ Rationalität : Sittlichkeit.

Beindruckend breit dagegen Albert 2010. 


\section{Philosophie und Theologie der reinen Gabe}

Anders verläuft die philosophische Debatte. Die von Jacques Derrida (1930-2004) ausgelöste Kontroverse um die (Un-)Möglichkeit der ,reinen“ Gabe (vgl. auch Hammerschmied 1998) ist hierbei wichtig. ${ }^{24}$ Aber die theologischen Wurzeln liegen tiefer, stellt die Gabe doch eine theologische (insb. in der Theologie [und in der theologischen Anthropologie] daher breit diskutierte: Lintner 2008) Ur-Kategorie dar (Ebner 2013).

Gar nicht einmal nur die Lutherische Tradition der Reziprozität, auch zu ihr gibt es neuere Literatur (Holm 2006; Holm/Widmann 2009), wäre anzuführen; eine breite religionsgeschichtliche und religionsphänomenologische Forschungstradition zum „do ut des“-Prinzip hat die Gabe-Gegengabe-Beziehung zwischen Gott und Mensch kult- und ritualtheoretisch (theoriegeschichtlich erfassbar in der Kontroverse um die berühmte Cambridge-Schule) herausgearbeitet. Die religionsphänomenologischen Klassiker ${ }^{25}$ will ich hier nur anführen. Die damit verbundene ausgeprägte kontroverse Dogmengeschichte (ich erinnere nur an das Werk von William Roberston Smith [1846-1894]: vgl. Maier 2009) möchte ich hier ebenfalls nur andeuten. Insofern ist die Gabe vor allem in opfertheoretischen Zusammenhängen (auch dazu ist die Literaturlage komplex: vgl. u. a. Vollmer 2009; Wolf 2004) breit erörtert worden, sowohl theologisch als auch allgemein religionswissenschaftlich, mit alt- wie mit neutestamentlichen und natürlich auch transeuropäischen und nicht nur orientalistischen Bezügen. Analysen zu Mahlgemeinschaften (Stein 2008), aus der Zeit, in der die Agape als Sättigungsmahl (hausgemeinschaftlich, im hellenistischen Vereinswesen kultgenossenschaftlicher Art eingebunden $)^{26}$ noch mit dem Herrenmahl vereint war und sich nicht, liturgiewissenschaftlich betrachtet, zur gottesdienstlichen Eucharistiefeier symbolisch verflüchtigte, und Tischgesellschaften (Därmann/Lemke 2008) vor allem im Typus der Tischgenossenschaften, von denen sogar Albert Hirschman (1915-2012) einmal in einem kleinen Essay gehandelt hat, stehen hier im Mittelpunkt der Erörterungen.

Bei mir ist die Mahlgemeinschaft mehr als eine Spende, die als Fremdhilfe an Dritte (wie im Fall des Fremdensozialrechts im Deuteronomium) darstellt:

$$
\mathrm{A} \rightarrow \mathrm{B} \text {. }
$$

Eine Mahlgemeinschaft ist eine Reziprozitätsbeziehung:

$$
\mathrm{A} \rightarrow \mathrm{B} \rightarrow \mathrm{A} \text {. }
$$

Ich stelle den Zusammenhang als Linie dar; an sich handelt es sich um einen Kreis. Die Differenz zwischen einer Gegenseitigkeitsgabe und einer Spende ist natürlich fließend, weil zwar $A \neq B$, aber A, B sind alles Mitglieder der Gemeinde Ĝ:

$$
\hat{\mathrm{G}}=\hat{\mathrm{G}}\{\mathrm{A}, \mathrm{B}, \mathrm{C}, \ldots\} .
$$

Damit ist auch (noch) nichts über die möglichen Formen der Asymmetrie und Symmetrie der Reziprozität ausgesagt, auch nicht über homo- oder heteromorphe Austauschrelationen:

homomorph bedeutet: $\left\{\varphi_{\mathrm{to}} \leftrightarrow \varphi_{\mathrm{t} 1}\right\}$,

heteromorph bedeutet: $\left\{\varphi_{\mathrm{to}} \leftrightarrow \omega_{\mathrm{t} 1}\right\}$,

24 Hier ist erwähnenswert Busch 2004.

25 Vgl. dazu das religionsphänomenologische Schrifttum von Geradus van der Leeuw (1890-1950) und Geo Widengren (1907-1996). Vgl. auch in Heiler 1921.

26 Von denen bereits Georg Heinrici (1844-1915) handelte. Vgl. auch Öhler 2005. 


$$
\text { wobei } \varphi \neq \omega \text {. }
$$

Hier liegt ein Spannungsfeld vor mit Blick auf die Transformation des ,parochialen Altruismus“ zur Universalität der Gabe als Liebesethik. In der älteren Forschung war dieses Problem aber bereits bekannt als Übergang vom Gruppenethos (der „,korporativen Persönlichkeit“, wie sie der Kulturanthropologie und historischen Psychologie des Alten, aber auch des Neuen Testaments zu entnehmen ist) zur personalen Ethik im Kontext von Insider-Outsider-Relationen (Schulz-Nieswandt 2012 b; 2013 e). An diesem Punkt schließt sich sodann eine breite neuere Gastfreundschaftskulturforschung (Liebsch 2008; Hiltbrunner 2005) an, die nicht weiter dargelegt werden soll. ${ }^{27}$ (Derrida hat auch den Diskurs zur Kategorie der Freundschaft angestoßen.) Hier gehört auch die ganze Empathie-Diskussion (aus Sicht der psychodynamischen Bindungsforschung) angesiedelt.

Die neuere theologische Gabe-Debatte (auch Hoffmann 2013) resultiert wohl vor allem aus der ethischen Relevanz der Kategorie ${ }^{28}$ und betont im Schnittbereich zur Ontologie und philosophischen Anthropologie (auch als Onto-Theologie) die Verbindungen zu vielfältigen neueren (auch hier wiederum oftmals französischen) Entwicklungslinien des phänomenologischen und hermeneutischen Denkens.

Zentral ist, und darauf möchte ich an dieser Stelle die (viel komplexere und stärker differenzierte/ verästelte ${ }^{29}$ Debatte verkürzen, hierbei der Beitrag und die (auch deutsche) Rezeption von JeanLuc Marion $\left({ }^{*} 1946\right) .{ }^{30}$ Es ist nicht falsch, diese (mitunter deutlich katholisch geprägte) Debatte um die Gabe (wie eine neuere einschlägige Literatur darlegen kann) als Philosophie der Liebe (Wolf 2006), spezifischer als Philosophie der Performativität, ja der Präsenz der Liebe als Ereignis für den Menschen in seiner Existenz (auch gerade als homo patiens) zu verstehen. Die Liebe wird zur Gabe, als Ruf eine An-Rufung des Menschen (Marion/Wohlmuth 2000), die ihn, wenn er (und gerade im „Verpassen“ liegt die Eigenschaft des homo abyssus [Ferdinand Ulrich] begründet) die Chance (eigentlich die Freiheit als „Zwang“ zum Wagnis des Seins [Gabriel Marcel; Peter Wust], weshalb der „Mut“ [Paul Tillich] als Antwort auf die Sorgestruktur der menschlichen Existenz [Ruffing 2011] so wichtig wird: Schulz-Nieswandt 2009) ergreift, selbst zur Liebe und somit zur Gabe befähigt und dergestalt gelingendes Dasein in seiner ganzen Gestaltqualität (Kuhr 2012) ermöglicht.

Herausgefordert ist die neuzeitliche Subjektphilosophie damit aus der Perspektive einer „Philosophie des Mich“" in der Sicht auf die dialogische Anrede (Schulz-Nieswandt 2010, S. 564ff.) als Ermöglichung von Personalität.

An Karl Löwith's (1897-1873) Sicht auf die Person in der - immer reziproken - existenziellen Rolle des Mitmenschen (Löwith 2013; dazu auch in Wojcieszuk 2010) wäre unbedingt zu erin-

27 Dazu etwa auch in sozialpolitisch adaptierter Form: Schulz-Nieswandt 2012.

28 Aus der neueren Literatur sei in philosophischer Perspektive auf Bauer 2012 verwiesen.

29 Ich verweise einerseits auf die personalistischen Strömungen in Philosophie und Theologie des 20. Jahrhunderts, eben aber auch uns insbesondere andererseits auf die jüdisch geprägten Beiträge bei Franz Rosenzweig (1886-1929), Martin Buber (1876-1965), Emmanuel Levinas (1905/06-1995). Zu Levinas im Zusammenhang mit dem von Derrida aufgeworfenen Frage nach der Möglichkeit einer unbedingten Gabe in Reinheitskultur vgl. auch Schulz-Nieswandt u. a. 2006, S. 173 f., FN 257.

30 Vgl. auch die Interpretationen zu Marion in Gondek/Tengelyi 2011: Vgl. ferner Gabel/Joas 2007 sowie GerlFalkovitz 2013. 
nern. Insofern ist die Person immer nur daseiend im Zwischenraum $\{\leftrightarrow\}$, der durch den Dialog (Heinze 2011) konstituiert ist.

Das in-die-Welt-Geworfen-Sein ist somit die immer seiende Einbettung, ohne die Personalisierung gar nicht möglich ist. Das ist das vorgängige Dritte, das Gemeinsame des sinnhaften Horizonts, vor dem sich Ich und Du in ihrer reziproken Gestaltwerdung zur Wahrheit der Existenz durchringend überhaupt erst verstehen lassen. In einem gewissen Sinne ist daher in der Sozialtheorie die kleinste Einheit der Analyse nicht das Individuum als Atom, sondern, existenzial gedacht, die Person (Schulz-Nieswandt 2011 a) in ihrer molekularen Figuration.

\section{Rechtsethnologie und die Genealogie der Gnade}

Die klassische Rechtsethnologie (wie bei Sebald Rudolf Steinmetz [1842-1940]) thematisierte der Tat und die Reaktion der Vergeltung als Reziprozität (Steinmetz 1928).

Im Ursprung ist Rechtsgeschichte hier in Religionsgeschichte eingebettet. Denn - wie im alttestamentlichen Kontext - sind Tun und Ergehen reziprok verkoppelt. Die Theologie des Altes Testaments hat dies im Rahmen eingehender Exegese dargelegt. Hier ist die Tat der Ruf, und das vom Vergelten geprägte Ergehen die Antwort. Und auch hier gibt es negative „Gabezyklen“: die Rache als eskalierende, weil kumulativ-zirkulative) Blutrache (Vendetta). Die Vergebung ist nun ein Akt Gottes, ein Walten des einen Gottes. Kontext ist die Königsprädikation Gottes in der nach-exilischen Zeit, in der die Sprache der zweiten Bundestheologie als einseitiger Gnaden-Kontrakt zwischen JHWE und seinem Volk geschlossen wurde. Und dieses Walten ist nicht identisch mit dem Verständnis des Schicksals bei Homer, wenngleich auch dort die Geschicke im Wechselspiel zwischen den Göttern und den Menschen bestimmt werden. Doch im archaischen Griechenland wurzelt jenes spezifische Verständnis von Tragik, in der der Mensch „,unschuldig schuldig“ wird (so die Formulierung von Wolfgang Schadewaldt im vierten Band seiner Tübinger Vorlesungen). Der daimon, der hier wirkt, ist psychologisch ganz anderer Art, als es die Dämonen sind, die im Zuge der Christianisierung als böse Geister immer des Teufels sind.

Im Beginn dieser Rechtsgeschichte, die zunächst in einfacher Apodiktik reguliert wurde, ist die Tat als Tabu-Verletzung forensisch, der soziale Raum ein forensischer Raum. Denn die Tat verletzt die Hygiene der Reinheitskultur der sozialen Beziehungen. So auch der Inzest (Trinkaus 2005). Im Kontext der Religionsgeschichte des altorientalischen Alten Testaments verschiebt sich nun die Vergeltung zur Vergebung. JHWE als Gott der Liebe erweist sich in seiner Gnade als Gabe.

Gnade ist jedoch die liebevolle Vergebung im Modus der Macht. Gnade setzt die asymmetrische Souveränität in der hierarchischen Figuration von Gott (als das/der ganz Andere der Unbedingtheit) und Mensch voraus. Die Haltung der Barmherzigkeit, ausgedrückt in der altorientalischen Metapher von Hirt und Herde (Schulz-Nieswandt 2010, S. 247, 261), wie sie sich u. a. christologisch seit der pharaonisch geprägten römischen Kaiserzeit fortsetzte, ist eine Haltung des liebenden Herzens, aber eben ein Erbarmen - „von oben nach unten“.

Eine kritische Genealogie der Gnade ist - im Sinne von Michel Foucaults (1926-1984) Studien zur Pastoralmacht - noch nicht (trotz Hardt 2005) erschöpfend geschrieben worden. Sie dürfte als 
kulturelle Tiefengrammatik auch aktueller Formen sozialer Praktiken des Helfens von hermeneutischer Bedeutung sein.

\section{Herrschaft als Reziprozität: Öffentliches Wirtschaften}

Auch der Staat funktioniert, finanzsoziologisch gesehen, als sozialer Rechtstaat im Modus einer - nun aber vertikalen - Reziprozität. Dies verweist auf die kulturgeschichtliche Genealogie der archetypischen (Schulz-Nieswandt 2000 a) Wurzeln im Sakralkönigtum (Schulz-Nieswandt 2003). Psychoanalytisch betrachtet haftet der Staatszentriertheit des modernen Menschen ein Restbestand infantiler Regression an, zu der ihn die Kontingenzproblematik seiner Existenz aus seiner Ur-Angst (Schulz-Nieswandt 2010 a; 2010 b; 2012 c), aber auch seines Ur-Vertrauens, in seinem psychodynamischen Drama treibt.

Doch ist dieser Staat wohl angesichts des Wachstums der sozialen Komplexität zwingend notwendig, da die Gestalt der genossenschaftlichen Gebilde nicht alle Probleme effektiv bewältigen kann. Im Kontext der kommunalen Daseinsvorsorge kann darin wohl noch ein lebensweltlich verankerbares Potenzial der Demokratisierung der öffentlichen Wirtschaft vermutet werden, also dort, wo es um das Bürgervermögen geht, dass nicht im Modus der Privatisierung ausverkauft werden sollte (Schulz-Nieswandt 2013 b). Die Engführung des Daseinsvorsorgestaates zum Gewährleistungsstaat (Schulz-Nieswandt 2012 a) in der Form eines kontraktuellen Pflichtenheftmanagers im obligatorischen Ausschreibungswettbewerb kommt dagegen einer Kastration gleich.

\section{Literaturverzeichnis}

Adloff, F. (2010): Philanthropisches Handeln. Frankfurt am Main-New York: Campus.

Adloff, F./Mau, St. (Hrsg.) (2005): Vom Geben und Nehmen. Frankfurt am Main-New York: Campus.

Albert, A. Chr. (2010): Helfen als Gabe und Gegenseitigkeit. Heidelberg: Winter.

Algazi, G./Groebner, V./Jussen, B. (Hrsg.) (2003): Negotiating the Gift. Pre-Modern Figurations of Exchange. Göttingen: Vandenhoeck \& Ruprecht.

Bähr, Chr. u. a. (Hrsg.) (2009): Überfluss und Überschreitung. Die kulturelle Praxis des Verausgabens. Bielefeld: transcript.

Bataille, G. (1985): Die Aufhebung der Ökonomie. Berlin: Matthes \& Seitz.

Baudrillard, J. (2011): Der symbolische Tausch und der Tod. Berlin: Matthes \& Seitz.

Bauer, K. (2012): Einander zu erkennen geben. Das Selbst zwischen Erkenntnis und Gabe. Freiburg i. Br.: Alber.

Berner, U./Bochinger, Chr./Flasche, R. (Hrsg.) (2005): Opfer und Gebet in den Religionen. Gütersloh: Gütersloher Verlagshaus.

Blome-Drees, J. (2011): Genossenschaftslehre. Berlin: LIT.

Brinkmann, V. (2010): Sozialwirtschaft. Wiesbaden: Gabler.

Busch, K. (2004): Geschickte Gaben. Aporien der Gabe bei Jacques Derrida. München: Fink.

Caillé, A. (2008): Anthropologie der Gabe. Frankfurt am Main-New York: Campus.

Därmann, I. (2010): Theorien der Gabe zur Einführung. Hamburg: Junius.

Därmann, I./Lemke, H. (2008): Die Tischgesellschaft. Bielefeld: transcript.

Davis, N. Z. (2002): Die schenkende Gesellschaft. Zur Kultur der französischen Renaissance. München: Beck.

Ebner, F. u. a. (Hrsg.) (2013): Geben und Nehmen. Jahrbuch für Biblische Theologie, Bd. 27. Neukirchen-Vluyn: Neukirchener.

Finley, M. I. (2005): Die Welt des Odysseus. Frankfurt am Main-New York: Campus.

Frank, E. M. (2012): Der Gabe auf der Spur. Eine soziologische Untersuchung zu Gabe und Geschenk im gesellschaftlichen Kontext. Frankfurt am Main: Lang.

Gabel, M./Joas, H. (Hrsg.) (2007): Von der Ursprünglichkeit der Gabe. Jean-Luc Marions Phänomenologie in der Diskussion. Freiburg i. Br.: Alber. 


\section{Frank Schulz-Nieswandt}

Gerl-Falkovitz, H.-B. (Hrsg.) (2013): Jean-Luc Marion. Studien zum Werk. Dresden: Text \& Dialog.

Gimesi, Th./Hanselitsch, W. (Hrsg.) (2010): Geben, Nehmen, Tauschen, Wien: LIT.

Görlich, J. (1992): Tausch als rationales Handeln. Zeremonieller Gabentausch und Tauschhandel im Hochland von Papua-Neuginea. Berlin: Reimer.

Gondek, H.-F./Tengelyi, L. (2011): Neue Phänomenologie in Frankreich. Frankfurt am Main: Suhrkamp.

Grund, A. (Hrsg.) (2014): Opfer, Geschenke, Almosen. Die Gabe in Religion und Gesellschaft. Stuttgart: Kohlhammer.

Hammerschmied, G. J. (1998): „Milde Gabe“. Bruchstücke einer Philosophie der Spender. Wien: Passagen.

Hardt, P. (2005): Genealogie der Gnade. Berlin: LIT.

Heiler, F. (1921): Das Gebet. Eine religionsgeschichtliche und religionspsychologische Untersuchung. 3. Aufl. München: Reinhardt.

Heinz, H. (1989): Kropotkin zur Einführung. Hamburg: Junius.

Heinze, E.-M. (2011): Einführung in das dialogische Denken. Freiburg i. Br.-München: Alber.

Hénaff, M. (2009): Der Preis der Wahrheit. Gabe, Geld und Philosophie. Frankfurt am Main: Suhrkamp.

Hénaff, M. (2014): Die Gabe der Philosophen. Bielefeld: transcript.

Hiltbrunner, O. (2005): Gastfreundschaft in der Antike und im frühen Christentum. Darmstadt: WBG.

Hobuß, St./Tams, N. (Hrsg.) (2014): Lassen und Tun. Kulturphilosophische Debatten zum Verhältnis von Gabe und kulturellen Praktiken. Bielefeld: transcript.

Hoffmann, T. J. (2009): Verschwendung. Philosophie, Soziologie und Ökonomie des Überflusses. Frankfurt am Main: Lang.

Hoffmann, V. (2013): Skizzen zu einer Theologie der Gabe. Freiburg i. Br.: Herder.

Holm, B. K. (2006): Gabe und Geben bei Luther. Das Verhältnis zwischen Reziprozität und reformatorischer Rechtfertigungslehre. Berlin-New York: de Gruyter.

Holm, B./Widmann, P. (Hrsg.) (2009): Word - Gift - Being. Tübingen: Mohr Siebeck.

Joas, H. (2014): Die Sakralität der Person. Eine neue Genealogie der Menschenrechte. Frankfurt am Main: Suhrkamp.

Kappeler, P./Schaik, C. P. (Hrsg.) (2005): Cooperation in Primates and Humans. Berlin: Springer.

Klein, St. (2010): Der Sinn des Gebens. Frankfurt am Main: S. Fischer.

Köstler, U. (2007): Aktivierung des Bürgers mittels Zeittauschsystemen, Seniorengenossenschaften sind mehr als nur Tauschringe. In: Zeitschrift für öffentliche und gemeinwirtschaftliche Unternehmen 2007 (4), 390-413.

Köstler, U. (2012): Nightline: Das Zuhör- und Informationstelefon von Studierenden für Studierende. Baden-Baden: Nomos.

Köstler, U. (2013): Sammelbesprechung. In: Zeitschrift für öffentliche und gemeinwirtschaftliche Unternehmen 36 (2-3), S. 225-227.

Köstler, U./Schulz-Nieswandt, F. (2010): Genossenschaftliche Selbsthilfe von Senioren. Motive und Handlungsmuster bürgerschaftlichen Engagements. Stuttgart: Kohlhammer.

Kuhr, I. (2012): Gabe und Gestalt. Theologische Phänomenologie bei Hans Urs von Balthasar. Regensburg: pustet.

Lang, H. (2010): Systeme der Wirtschaftsethnologie. Berlin: Reimer.

Laux, B. (Hrsg.) (2013): Heiligkeit und Menschenwürde. Hans Joas‘ neue Genealogie der Menschenrechte im theologischen Gespräch. Freiburg i. Br.: Herder.

Liebersohn, H. (2011): The Return of the Gift. European History of a Global Idea. New York: Cambridge University Press.

Liebsch, B. (2008): Für eine Kultur der Gastlichkeit. Freiburg i. Br.: Alber.

Lintner, M. M. (2008): Eine Ethik des Schenkens. Wien-Berlin: LIT.

Löwith, K. (2013): Das Individuum in der Rolle des Mitmenschen. Freiburg i. Br.: Alber.

Maier, B. (2009): William Robertson Smith. His Life, his Work and his Times. Tübingen: Mohr Siebeck.

Maio, G. (Hrsg.) (2013): Ethik der Gabe. Humane Medizin zwischen Leistungserbringung und Sorge um die Anderen. Freiburg i. Br.: Herder.

Marion, J.-L./Wohlmuth, J. (2000): Ruf und Gabe. Zum Verhältnis von Phänomenologie und Theologie. Alfter: Borengässer.

Mein, G./Schößler, F. (Hrsg.) (2005): Tauschprozesse. Kulturwissenschaftliche Verhandlungen des Ökonomischen. Bielefeld: transcript.

Miklautz, E. (2010): Geschenkt. Tausch gegen Gabe - eine Kritik der symbolischen Ökonomie. München: Fink.

Moebius, St. (2006): Marcel Mauss. Konstanz: UVK.

Moebius, St. (2006 a): Die Zauberlehrlinge. Soziologiegeschichte des Collège de Sociologie (1937-1939). Konstanz: UVK.

Moebius, S. (2010): Von Mauss zu Hénaff: Eine kleine Wirkungsgeschichte des Essai sur le don. In: WestEnd. Neue Zeitschrift für Sozialforschung 7 (1), S. 68-80.

Moebius, St./Papiloud, Chr. (Hrsg.) (2006): Gift - Marcel Mauss` Kulturtheorie der gabe. Wiesbaden: VS Verlag für Sozialwissenschaften.

Mohr, S./Ouart, L.-M./Vetter, A. (Hrsg.) (2013): (aus)tauschen. Erkundungen einer Praxisform. Berlin: Panama.

Motakef, M. (2011): Körper Gabe. Ambivalente Ökonomien der Organspende. Bielefeld: transcript.

Negel, J. (2005): Ambivalentes Opfer. Paderborn u. a.: Schöningh.

Öhler, M. (2005): Die Jerusalemer Urgemeinde im Spiegel des antiken Vereinswesens. In: NTS 51, S. 393-415. 


\section{M.A.U.S.S. spielen! Die Gabe im multi-disziplinären Blick}

Preissing, S. (2009): Tauschen - Schenken - Geld? Ökonomische und gesellschaftliche Gegenentwürfe. Berlin: Reimer.

Pulcini, E. (2004): Das Individuum ohne Leidenschaften. Berlin: Diaphanes.

Reichlin, S. (2009): Ökonomien des Begehrens, Ökonomie des Erzählens. Zur poetologischen Dimension des Tauschens in Mären. Göttingen: Vandenhoeck \& Ruprecht.

Rössler, M. (2005): Wirtschaftsethnologie. Berlin: Reimer.

Ruffing, R. (2011): Der Sinn der Sorge. Freiburg i. Br.: Alber.

Scheuregger, D. u. a. (Hrsg.) (2007): Reziprozität und Wohlfahrtsstaat. Analysepotenzial und sozialpolitische Relevanz. Opladen-Farmington Hills: Barbara Budrich.

Schulz-Nieswandt, F. (2000): Gilden als ,totales soziales Phänomen“ im Mittelalter. Weiden-Regensburg: eurotrans.

Schulz-Nieswandt, F. (2000 a): Zur einer archetypischen Morphologie der Sozialpolitik. Genossenschaft und sakrales Königtum. Weiden-Regensburg: eurotrans.

Schulz-Nieswandt, F. (2001): Die Gabe. Der gemeinsame Ursprung der Gesellung und des Teilens im religiösen Opferkult und in der Mahlgemeinschaft. In: Zeitschrift für Sozialreform 47 (1), S. 75-92.

Schulz-Nieswandt, F. (2001 a). Der Potlatsch: Sozialpathologie des Agonalen oder Übergangsphänomen der Gabe zum vertikalen Ressourcenpooling? In ders. u. a. (Hrsg.): Einzelwirtschaften und Sozialpolitik zwischen Markt und Staat in Industrie- und Entwicklungsländern Festschrift für Werner Wilhelm Engelhardt zum 75. Geburtstag. Marburg: Metropolis Verlag, S. 99-108.

Schulz-Nieswandt, F. (2002): Zur Genossenschaftsartigkeit der Gesetzlichen Krankenversicherung. Weiden-Regensburg: eurotrans.

Schulz-Nieswandt, F. (2003): Herrschaft und Genossenschaft. Berlin: Duncker \& Humblot.

Schulz-Nieswandt, F. (2006): Die Unbedingtheit der Gabeethik und die Profanität der Gegenseitigkeitsökonomik. Die genossenschaftliche Betriebsform als Entfaltungskontext der menschlichen Persönlichkeit im Lichte einer Form-Inhalts-Metaphysik. In: Rösner, H. J./Schulz-Nieswandt, F. (Hrsg.): Zur Relevanz des genossenschaftswissenschaftlichen Selbsthilfegedankens. Münster: LIT, 57-92.

Schulz-Nieswandt, F. (2006 a): Sozialpolitik und Alter. Stuttgart: Kohlhammer.

Schulz-Nieswandt, F. (2007): Kulturelle Ökonomik des Alterns - Zum Umgang mit dem Alter im Generationengefüge zwischen archetypischer Ethik und Knappheitsökonomik. In: Teising, M. u. a. (Hrsg.): Alt und psychisch krank. Stuttgart: Kohlhammer, S. 31-54.

Schulz-Nieswandt, F. (2007): Zur Relevanz des betriebsmorphologischen Denkens. Versuch einer sozialontologischen und anthropologischen Grundlegung. In: Bräunig, D./Greiling, D. (Hrsg.): Stand und Perspektiven der Öffentlichen Betriebswirtschaftslehre II. FS für Prof. Dr. Dr. h. c. mult. Peter Eichhorn anlässlich seiner Emeritierung. Berlin: BWV, S. 58-67.

Schulz-Nieswandt, F: (2007 a): Lebenslauforientierte Sozialpolitikforschung, Gerontologie und philosophische Anthropologie. Schnittflächen und mögliche Theorieklammern. In: Wahl, H.-W./Mollenkopf, H. (Hrsg.): Alternsforschung am Beginn des 21. Jahrhunderts. Berlin: AKA, S. 61-81.

Schulz-Nieswandt, F. (2008): Alter und Lebenslauf. Ein Beitrag zur philosophischen Anthropologie in sozialpolitischer Absicht. In: Aner, K./Karl, U. (Hrsg.): Lebensalter und Soziale Arbeit: Ältere und alte Menschen. Hohengehren: Schneider, S. 77-91.

Schulz-Nieswandt, F. (2009): Paul Tillichs Onto(theo)logie der Daseinsbewältigung und die Fundierung der Wissenschaft von der Sozialpolitik. In: Danz, Chr./Schüßler, W./Sturm, E. (Hrsg.): Religion und Politik. Internationales Jahrbuch für die Tillich-Forschung. Bd. 4. Berlin: LIT-Verlag, S. 125-138.

Schulz-Nieswandt, F. (2010): Wandel der Medizinkultur? Berlin: Duncker \& Humblot.

Schulz-Nieswandt, F. (2010 a): Öffentliche Daseinsvorsorge und Existentialismus. Eine gouvernementale Analyse unter besonderer Berücksichtigung der Wasserversorgung. Baden-Baden: Nomos.

Schulz-Nieswandt, F. (2010 b): Daseinsvorsorge und existenzielle Angst des Menschen. In: Jens, U./Romahn, H. (Hrsg.): Methodenpluralismus in den Wirtschaftswissenschaften. Marburg: Metropolis, S. 213-45.

Schulz-Nieswandt, F. (2011): Gesundheitsselbsthilfegruppen und ihre Selbsthilfeorganisationen in Deutschland. Der Stand der Forschung im Lichte der Kölner Wissenschaft von der Sozialpolitik und des Genossenschaftswesens. Baden-Baden: Nomos.

Schulz-Nieswandt, F. (2011 a): Bemerkungen zur gemeinwirtschaftlichen, einschließlich genossenschaftlichen Einzelwirtschaftslehre und Sozialwissenschaft im System der Wissenschaft von der Gesellschaftsgestaltungspolitik. Werner Wilhelm Engelhardt zum 85. Geburtstag. In: Zeitschrift für öffentliche und gemeinwirtschaftliche Unternehmen 34 (1), S. 100-109; wiederabgedruckt in Engelhardt, W. W. (2013): Beiträge zur Ordnungstheorie und Ordnungspolitik zwischen Markt und Staat. Von J. H. von Thünens Arbeiten her analysiert. Berlin: Duncker \& Humblot.

Schulz-Nieswandt, F. (2011 b): Das Altern im Kontext einer allgemeinen Theorie der Sozialpolitik. In: Stadt Frankfurt am Main (Hrsg.): Lebenslagen älterer Menschen. Ein Rückblick auf die Aktionswochen „Älter werden in Frankfurt“. Frankfurt a. M.: Societäts-Verlag, S. 10-34.

Schulz-Nieswandt, F. (2011 c): Berufsgenossenschaften und Europarecht. Eine sozialökonomische Analyse. Berlin: Duncker \& Humblot.

Schulz-Nieswandt, F. (2012): Gemeinschaftliches Wohnen im Alter in der Kommune. Das Problem der kommunalen Gastfreundschaftskultur gegenüber dem homo patiens. Berlin: Duncker \& Humblot. 


\section{Frank Schulz-Nieswandt}

Schulz-Nieswandt, F. (2012 a): „Europäisierung“ der Sozialpolitik und der sozialen Daseinsvorsorge? Eine kultursoziologische Analyse der Genese einer solidarischen Rechtsgenossenschaft. Berlin: Duncker \& Humblot.

Schulz-Nieswandt, F. (2012 b): Der homo patiens als Outsider der Gemeinde. Zur kulturellen und seelischen Grammatik der Ausgrenzung des Dämonischen. In: Zeitschrift für Gerontologie und Geriatrie 45 (7), S. 593-602.

Schulz-Nieswandt, F. (2012 c): Institutionelle Präferenzen der Bürger hinsichtlich der Erstellung kommunaler Daseinsvorsorgegüter - Eine tiefenpsychologische Re-Interpretation quantitativer Befragungsdaten. In: Schaefer, Chr./Theuvsen, L. (Hrsg.): Renaissance öffentlicher Wirtschaft. Baden-Baden: Nomos, S. 119-142.

Schulz-Nieswandt, F. (2012 d): Multi-disziplinärer Blick auf soziale gegenseitige Selbsthilfe und politische Selbstorganisation von Menschen mit chronischen und seltenen Erkrankungen. In: Monitor Versorgungsforschung 5 (1), S. 38-42.

Schulz-Nieswandt, F. (2013): Der leidende Mensch in der Gemeinde als Hilfe- und Rechtsgenossenschaft. Berlin: Duncker \& Humblot.

Schulz-Nieswandt, F. (2013 a): Der inklusive Sozialraum. Psychodynamik und kulturelle Grammatik eines sozialen Lernprozesses. Baden-Baden: Nomos.

Schulz-Nieswandt, F. (2013 b): Das Privatisierungs-Dispositiv der EU-Kommission. Das ontologische Existenzial der Daseinsvorsorge, die sakrale Doxa des Binnenmarktes und die „kafkaistischen“ Epiphanien der Regulationskultur. Berlin: Duncker \& Humblot.

Schulz-Nieswandt, F. (2013 c): Zur Implementation von innovativen Pilotprojekten in der Versorgungs- und Wohnlandschaft älterer Menschen: kulturelle Grammatik und systemische Choreographie. In: Karl, F. (Hrsg.): Transnational und translational - Aktuelle Themen der Alternswissenschaften, Reihe Soziale Gerontologie Bd. 3, Berlin: LIT, S. 97-118.

Schulz-Nieswandt, F. (2013 d): Transsektorale Integrationsversorgung als Problem des Gestaltwandels der Kultur professioneller Handlungsskripte - eine Mehr-Ebenen-Analyse. In: Haller, M./Meyer-Wolters, H./Schulz-Nieswandt, F. (Hrsg.). Alterswelt und institutionelle Strukturen. Würzburg: Königshausen \& Neumann, S. 153-168.

Schulz-Nieswandt, F. (2013 e): Wohnen im Alter in der Gemeinde - zwingende Gründe und kulturelle Barrieren der De-Institutionalisierung. In: informationsdienst altersfragen 40 (4), S. 9-15.

Schulz-Nieswandt, F. (2013 f): Neue Wege der Hauswirtschaft. Engagement in der Hilfe für pflegebedürftige und behinderte Menschen als professionelle Chance und ethische Herausforderung. In: Hauswirtschaft und Wissenschaft 61 (3), S. 135-141.

Schulz-Nieswandt, F./Köstler, U. (2011): Bürgerschaftliches Engagement im Alter. Stuttgart: Kohlhammer.

Schulz-Nieswandt, F./Köstler, U. (2012): Sparkassen und Kreditgenossenschaften - zwei ungleiche Zwillinge?! Ein morphologischer Beitrag zur widmungswirtschaftlichen Problematik öffentlicher oder gemeinwirtschaftlicher Unternehmen. In: Zeitschrift für öffentliche und gemeinwirtschaftliche Unternehmen 35 (3), S. 345-357.

Schulz-Nieswandt, Frank/Köstler, U. (2012 a): Das institutionelle und funktionale Gefüge von kommunaler Daseinsvorsorge und bürgerschaftlichem Engagement. Ein anthropologischer Zugang zu einem sozialmorphologisch komplexen Feld in sozialpolitischer Absicht. In: Zeitschrift für öffentliche und gemeinwirtschaftliche Unternehmen 35 (4), S. 465-478.

Schulz-Nieswandt, F./Köstler, U./Langenhorst, F./Marks, H. (2012): Neue Wohnformen im Alter. Wohngemeinschaften und Mehrgenerationenhäuser. Stuttgart: Kohlhammer.

Schulz-Nieswandt, F./Kurscheid, C. (2007): Die Schuld an der Schuld. Hamburg: Merus.

Schulz-Nieswandt, F./Sauer, M. (2010): Qualitative Sozialforschung in der Gerontologie - forschungsstrategische Überlegungen und implizite Anthropologie in der Gegenstandsbestimmung. In: Meyer-Wolters, H./Breinbauer, I.-M./Ferring, D./Haller, M. (Hrsg.). Transdisziplinäre Alternsstudien. Gegenstände und Methoden. Würzburg: Königshausen \& Neumann, S. 93-117.

Schulz-Nieswandt, F. u. a. (2006): Zur Genese des europäischen Sozialbürgers im Lichte der neueren EU-Rechtsentwicklung. Berlin: LIT.

Schulz-Nieswandt, F. u. a. (2009): Generationenbeziehungen. Netzwerke zwischen Gabebereitschaft und Gegenseitigkeitsprinzip. Berlin: LIT.

Starobinski, J. (1994): Gute Gaben, schlimme Gaben: die Ambivalenz sozialer Gesten. Frankfurt am Main: Fisch.

Stegbauer, Chr./Häußling, R. (Hrsg.) (2010): Handbuch Netzwerkforschung. Wiesbaden: VS Verlag für Sozialwissenschaften.

Stein, H. J. (2008): Frühchristliche Mahlfeiern. Tübingen: Mohr Siebeck.

Steinmetz, S. R. ((1928): Ethnologische Studien zur ersten Entwicklung der Strafe nebst einer psychologischen Untersuchung über Grausamkeit und Rachsucht. Bd. 1. Groningen: P. Noordhof.

Stephan, M. (2010): Geschenkt! Vom Schenken und seinen Zwängen in der Konsumgesellschaft. Marburg: Tectum. Suber, D. (2011): Émile Durkheim. Konstanz: UVK.

Thiel, Chr. (2011): Das ,bessere‘ Geld. Eine ethnographische Studie über Regionalwährungen. Wiesbaden: VS Verlag für Sozialwissenschaften.

Trinkaus, St. (2005): Blank Spaces. Gabe und Inzest als Figuren des Ursprungs von Kultur. Bielefeld: transcript.

Voland, E. (2013): Soziobiologie. 4., überarb. u. aktual Aufl. Berlin: Springer.

Vollmer, Th. (2009): Das Heilige und das Opfer. Wiesbaden: VS Verlag für Sozialwissenschaften.

Wagner, S. (2008): Lokale Tauschnetze. Wiesbaden: VS Verlag für Sozialwissenschaften. 


\section{M.A.U.S.S. spielen! Die Gabe im multi-disziplinären Blick}

Wagner-Hasel, B. (2000): Der Stoff der Gaben. Kultur und Politik des Schenkens und Tauschens im archaischen Griechenland. Frankfurt am Main-New York: Campus.

Wörer, S. (2012): Politik und Kultur der Gabe. Annäherung aus patriarchatskritischer Sicht. Frankfurt am Main: Lang.

Wojcieszuk, M. (2010): Der Mensch wird am Du zum Ich. Freiburg i. Br.: Alber.

Wolf, B. (2004): Die Sorge des Souveräns. Eine Diskursgeschichte des Opfers. Zürich: Diaphanes.

Wolf, K. (2006): Philosophie der Gabe. Mediationen über die Liebe in der französischen Gegenwartsphilosophie. Stuttgart: Kohlhammer.

Znoj, H. (1995): Tausch und Geld in Zentralsumatra. Berlin: Reimer. 